\title{
FAILURE EVOLUTION IN GRANULAR MATERIAL RETAINED BY RIGID WALL IN ACTIVE MODE
}

\author{
MAgDALENA PiETRZAK \\ Koszalin University of Technology \\ DANUTA LEŚNIEWSKA \\ Koszalin University of Technology, \\ Institute of Hydro-Engineering, Polish Academy of Sciences
}

\begin{abstract}
This paper presents a detailed study of a selected small scale model test, performed on a sample of surrogate granular material, retained by a rigid wall (typical geotechnical problem of earth thrust on a retaining wall). The experimental data presented in this paper show that the deformation of granular sample behind retaining wall can undergo some cyclic changes. The nature of these cycles is not clear - it is probably related to some micromechanical features of granular materials, which are recently extensively studied in many research centers in the world. Employing very precise DIC (PIV) method can help to relate micro and macro-scale behavior of granular materials.
\end{abstract}

\section{INTRODUCTION}

This paper presents a detailed study of a selected small scale model test, performed on a sample of surrogate granular material, retained by a rigid wall (typical geotechnical problem of earth thrust on a retaining wall). The model wall translated horizontally in an active mode (out of the granular sample).

The reason for presenting the test in such a way is that several unique observations on evolution of failure mechanism were made within the granular sample, which seems not to have been described earlier in literature. The observations were confirmed in a number of similar tests. The new findings were possible to be made due to employing image analysis, namely DIC (PIV) in elaboration of test results.

\section{MODEL TESTS}

The experimental set-up applied in this work was described in detail in [1]-[6]. Small-scale tests on granular samples retained by a movable rigid wall were performed in a glass-sided box (Fig. 1). The glass sides were $20 \mathrm{~mm}$ thick and loaded by lateral pressures from the granular material. The particular configuration included a smooth and rigid vertical wall, $180 \mathrm{~mm}$ high, supported by rods that were able to slide horizontally through the box. An active earth pressure mode was investigated, where 
a retaining wall moved away from the backfill. The mode of the test was quasi static with a constant wall displacement increment equal to $0.0625 \mathrm{~mm}(1 / 20$ of the supporting screw lead).

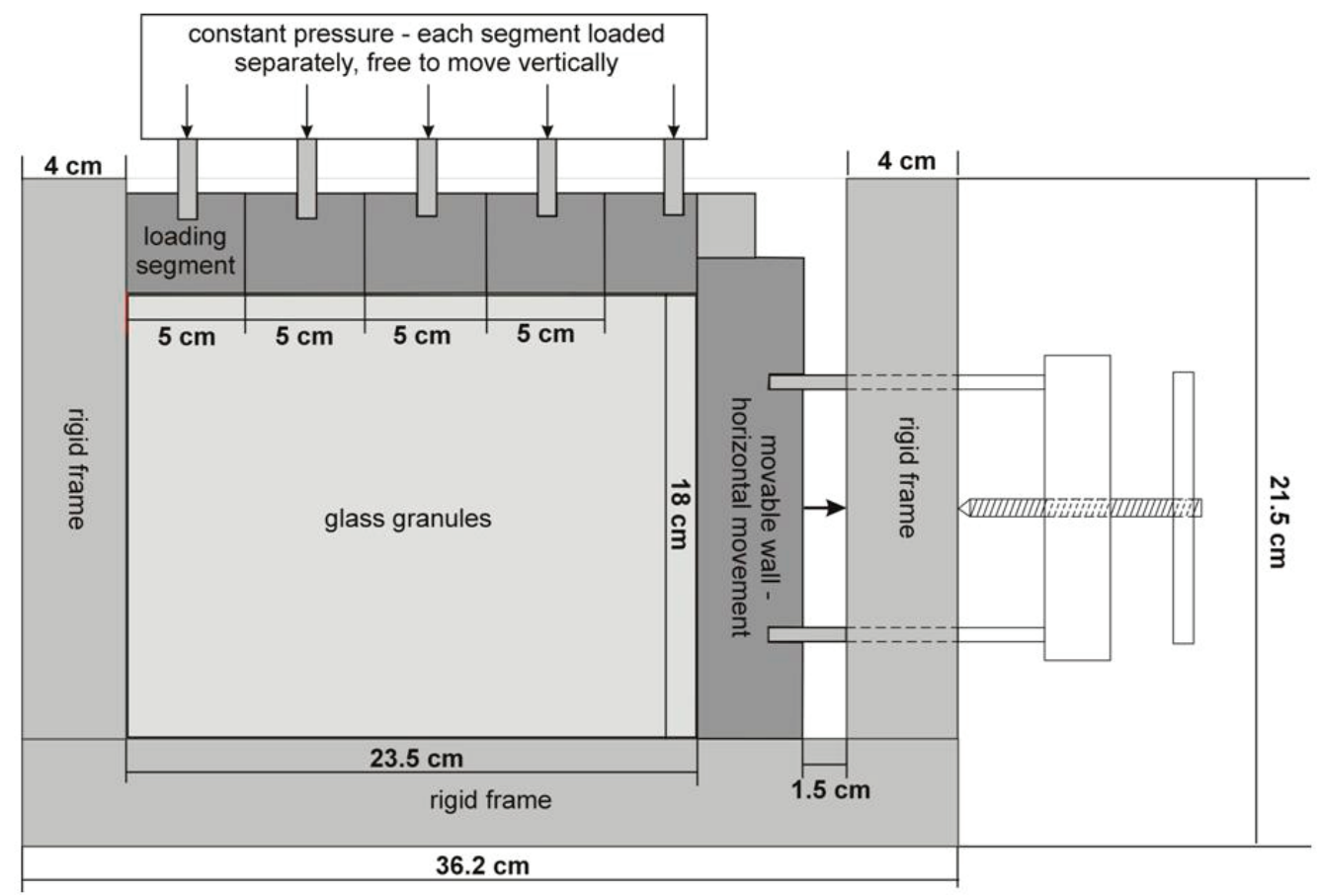

Fig. 1. Test layout

The tests were recorded with standard digital camera. Photographs were taken at each wall displacement step. Experimental displacement fields were determined on their base and hence strains calculated, using 2D digital image correlation (particle image velocimetry) DIC (PIV). Image analysis software geoPIV, elaborated by White and Take [7]-[9] was employed.

The tests were so-called "constrained" tests, during which the surface loading was provided by a footing formed of five rigid segments connected to the common pressure source, so that the pressure on each segment could be maintained as the footing articulated and settled (Fig. 1). The loading and wall displacements applied in the course of the test discussed in this paper (07_05_28) are shown in Fig. 2. The granular sample was loaded up to $1.6 \mathrm{MPa}$ in eight steps by $0.2 \mathrm{MPa}$. At pressure equal to 1.6 $\mathrm{MPa}$, the wall was permitted to move "actively" (out of the specimen) by releasing the support screw in constant increments. 


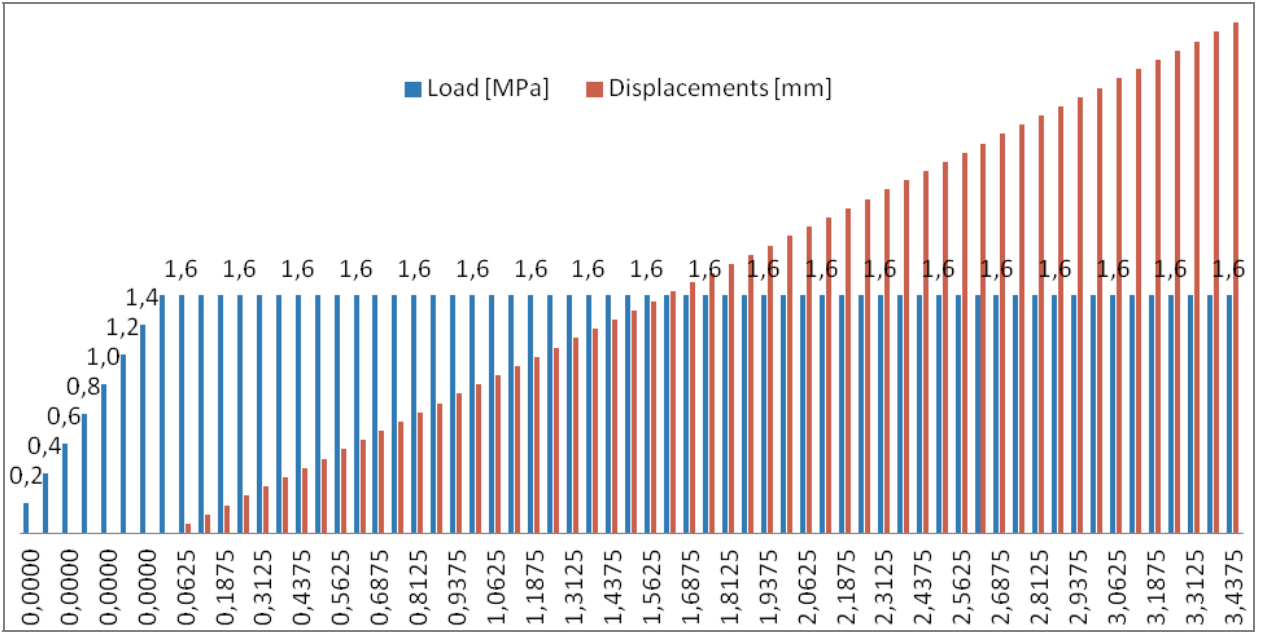

Fig. 2. Loading and wall displacement conditions during test $07 \_05 \_28$

\section{MATERIALS}

Starlitbeads 1000 spherical glass granules were used to form a granular sample $\left(d_{50}=1.1 \mathrm{~mm}\right)$. Only dense samples were investigated. To produce a dense sample, granular material was rained into the box from a hopper. The glass granules were selected to represent soil due to their transparency. The tests were registered both in ordinary and polarized light, to give not only strain, but also stress information based on photo-elasticity, but the latter is not a subject of this paper. Due to the demands of photo-elastic method the granular specimen was saturated with clove oil, having the same refraction index as glass [1]-[4].

\section{DIC (PIV) FOR GRANULAR MATERIALS}

Particle image velocimetry is a well-known velocity measuring non-invasive procedure, originally developed for fluid mechanics and used for the analysis of displacements in tests on soil models. It operates by tracking spatial variations of brightness within an image (divided into a mesh of PIV patches) by comparing successive images so that displacement data can be extracted from sequences of images, and strains then calculated from gradients of measured displacements. The geoPIV software developed by White and Take [7]-[9] for granular materials was employed in this study. The first step in geoPIV application is to divide the digital image into square patches of pixels forming a regular mesh - the extremes of the mesh coarseness were discussed by Leśniewska and Muir Wood in [3]. According to White et al. [8], 
the precision of PIV is a strong function of the patch size that is to be compared from image to image and is also influenced by the image content. If the patch is too small, then the software may not be able to recognize the displaced patch. A finer grid provides more details but also a greater potential for random data fluctuations. A trial and error method indicated that a reliable result (a displacement field containing no spurious vectors) could be obtained by using the mesh of square $40 \times 40$ pixel patches.

\section{DISCUSSION OF THE MODEL TEST RESULTS}

The direct result of the DIC (PIV) analysis is a displacement field, on the base of which different strain maps can be calculated by geoPIV software. Shear and volumetric strain maps obtained for the test 07_05_28 are presented in Figs. 3-8 and 11.

Figure 3 shows shear and volumetric strains which occurred after external loading rise from 1.4 $\mathrm{MPa}$ to its maximum value $1.6 \mathrm{MPa}$, just before applying the first wall displacement. It is interesting to notice that both shear and volumetric strains are not uniformly distributed, but form distinct, roughly parallel bands. As red colour in volumetric strains graphs indicates compression and blue colour dilation, we could call these bands "compaction bands", as compaction prevails over dilation in Fig. $3 \mathrm{~b}$. Similar bands are visible within shear strain map (Fig. 3a).

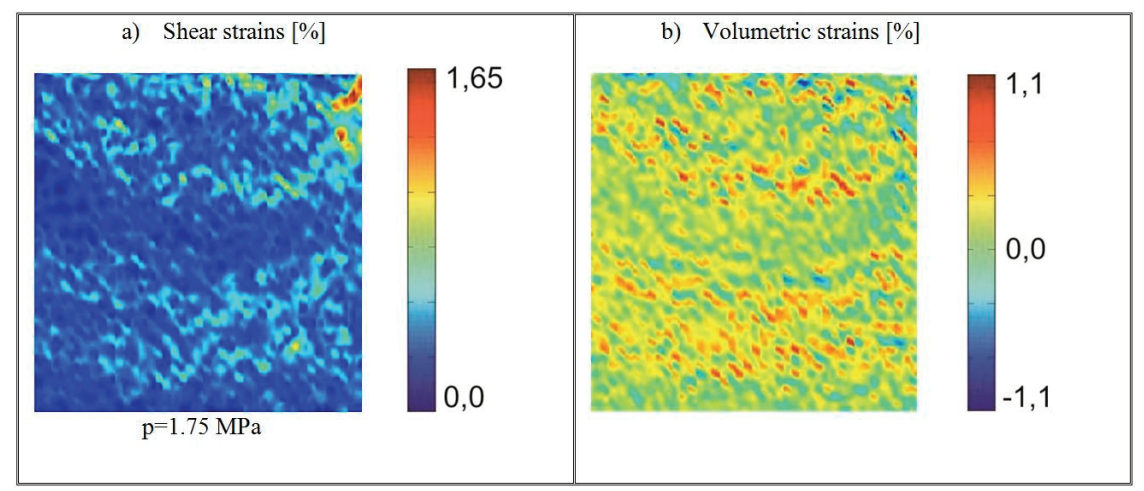

Fig. 3. "Compaction bands" due to external loading of the granular sample

Figures 4-8 represent the part of the test between the 26th and 31th wall displacement (Fig. 2). They show some characteristic short sequence, consisting of 5 events:

- One wall displacement increment resulting in strongly localized shear band, which cuts off slightly curvilinear wedge of much less deformed granular material (Fig. 4). This event of the sequence is characterized by relatively high shear strains (maximum 6.1\%). Figure $4 \mathrm{~b}$ shows that dilation prevails within shear band, while the inside of the cut-off wedge is clearly under compression, which must be the reason for its increased rigidity. 
- Three wall displacement increments resulting in diffused shear and volumetric strains inside the formerly less deformed wedge (Figs. 5-7). The maximum value of shear strains is similar at each displacement step (1.0, 1.1 and 1.2\%), suggesting some increase with the displacement value. Figures 5-7 suggest also that dilation prevails over compression within the uniformly deforming wedges.

- Final event of the sequence: single wall displacement resulting in localized shear band and again producing less deformed wedge outlined by it. Maximum shear strains experience rapid jump if compared to the proceding displacement steps (to 5.6\%). The situation resembles that of point 1 (beginning of the sequence) and is actually the end step of the characteristic "cycle". The inside of the wedge outlined by the localized shear band is mostly in compression (Fig. 8), which makes it more rigid than the surrounding granular material.

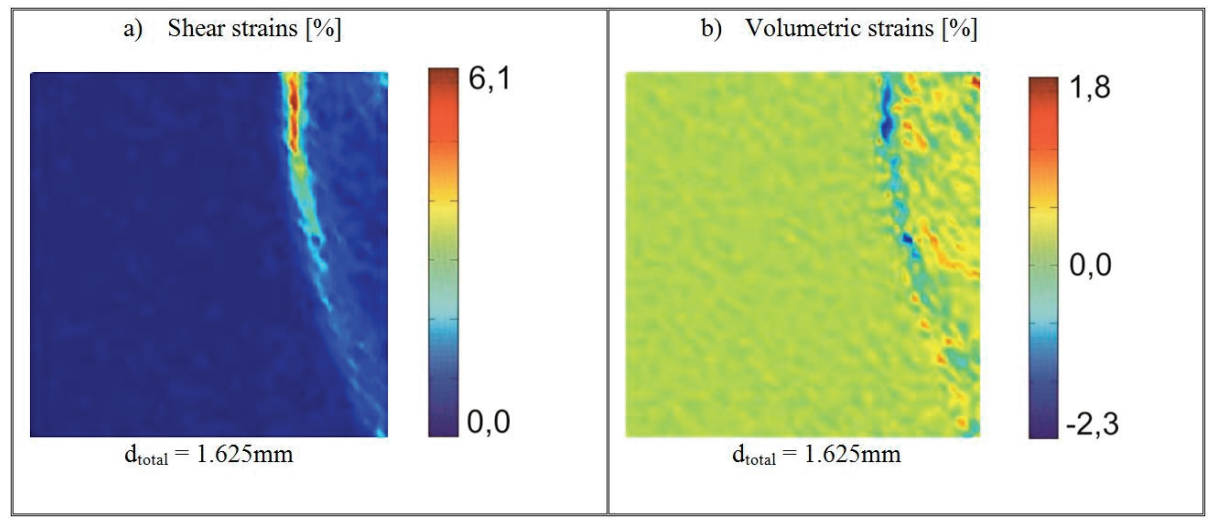

Fig. 4. Evolution of strains during test 07 05_28 - beginning of the deformation cycle - failure mechanism in a form of strongly localized shear band

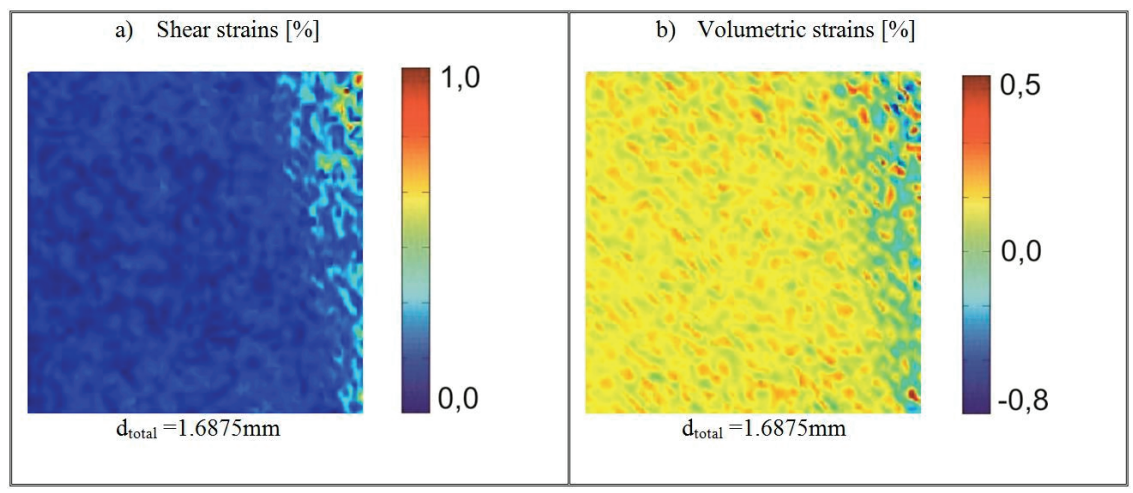

Fig. 5. Evolution of strains during test 07_05_28 - the first of intermediate events of the deformation cycle - diffused shear and volumetric strains appearing inside the formerly less deformed "wedge" 


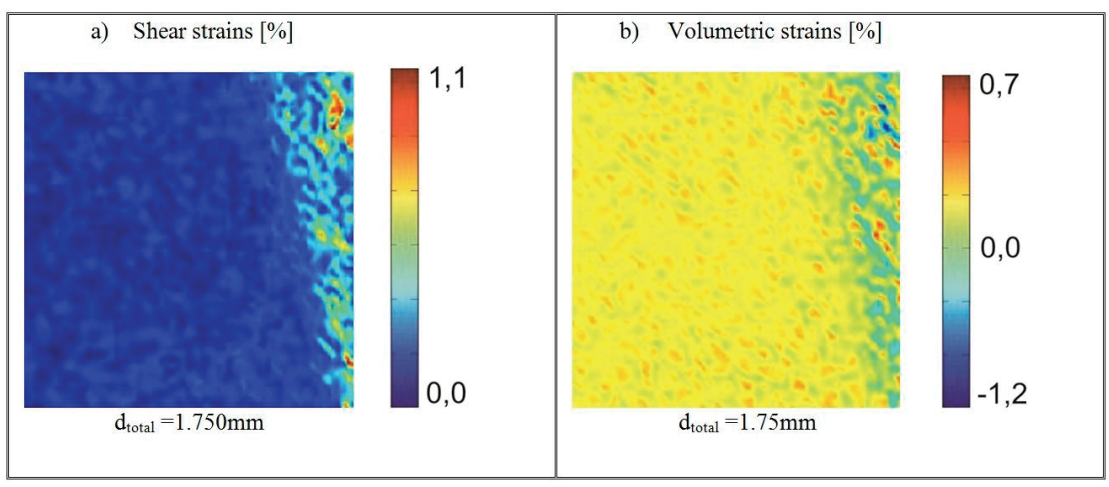

Fig. 6. Evolution of strains during test $07 \_05 \_28$ - the second of intermediate events of the deformation cycle - diffused shear and volumetric strains appearing inside the formerly less deformed "wedge"

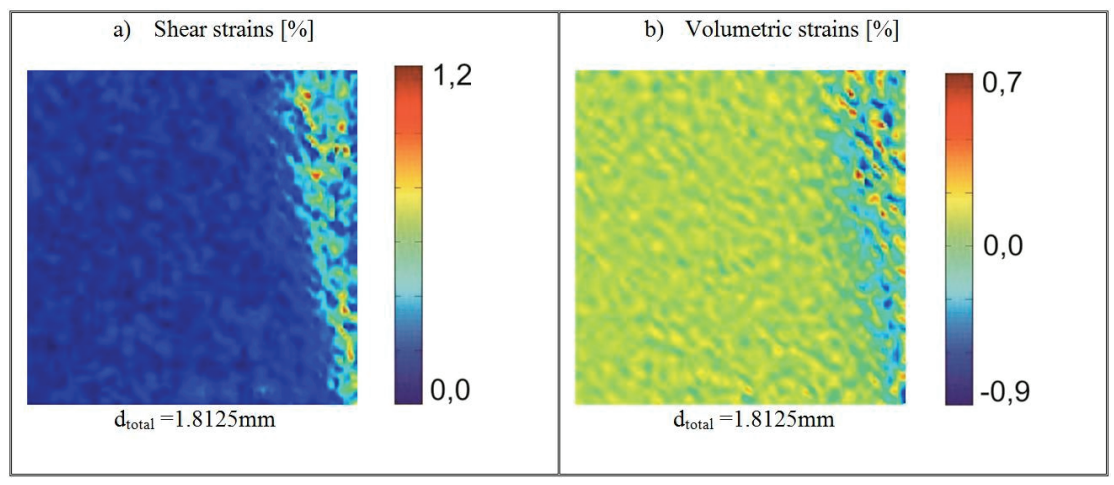

Fig. 7. Evolution of strains during test $07 \_05 \_28$ - the third of intermediate events of the deformation cycle - diffused shear and volumetric strains appearing inside the formerly less deformed "wedge"

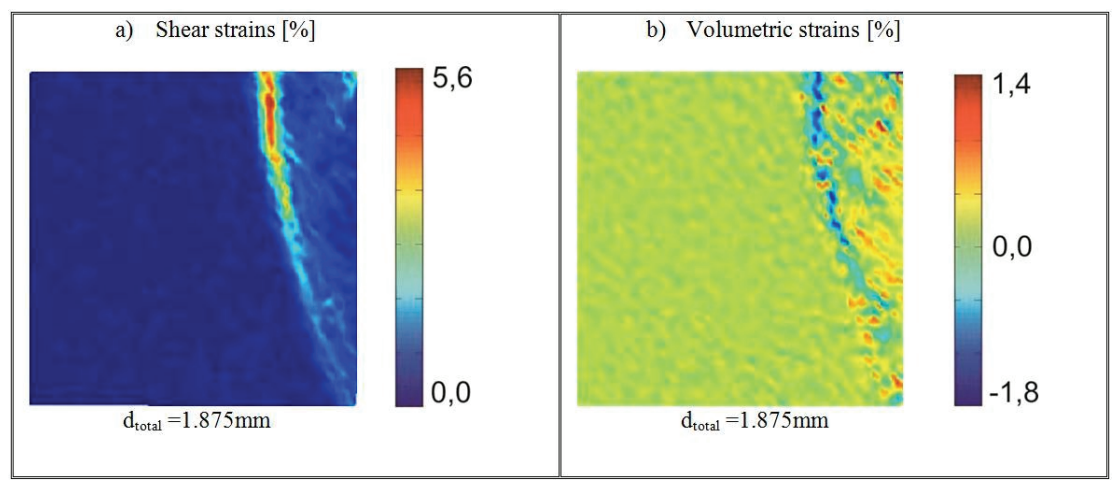

Fig. 8. Evolution of strains during test $07 \_05 \_28$ - end of the deformation cycle - failure mechanism in a form of strongly localized shear band and beginning of the new cycle 


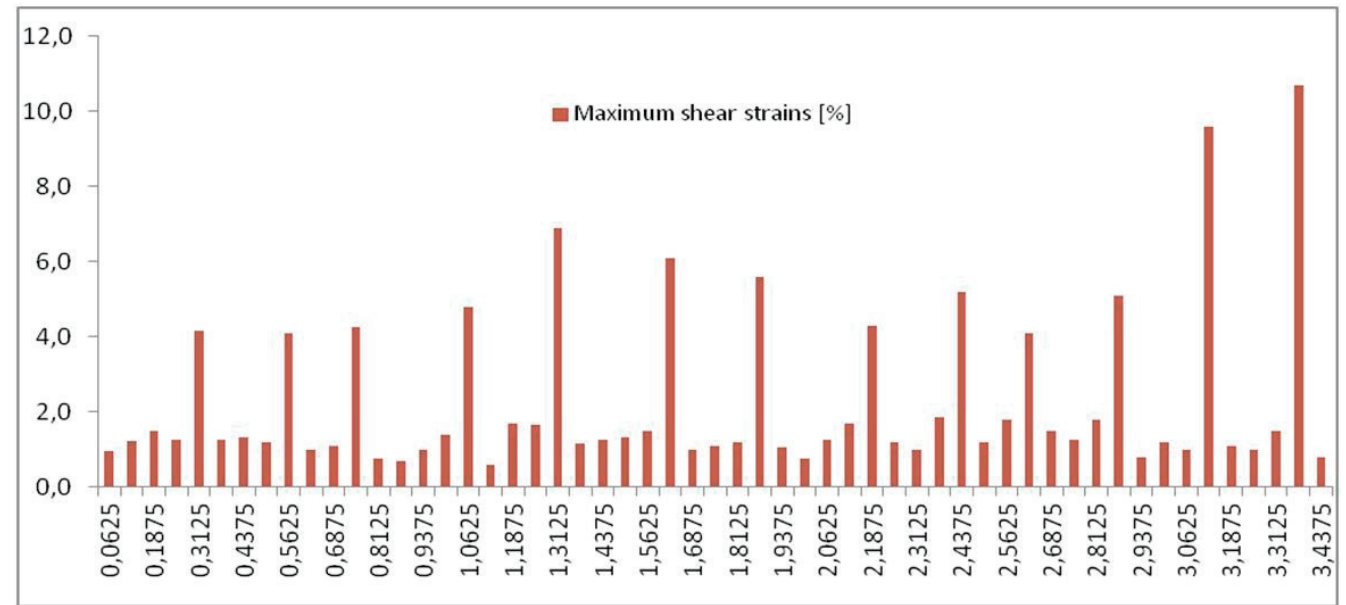

Fig. 9. Deformation cycles for the test on rigid wall, moving out of the granular material, visible in maximum shear strain values (vertical axis) observed for different wall displacements in $[\mathrm{mm}]$ (horizontal axis)

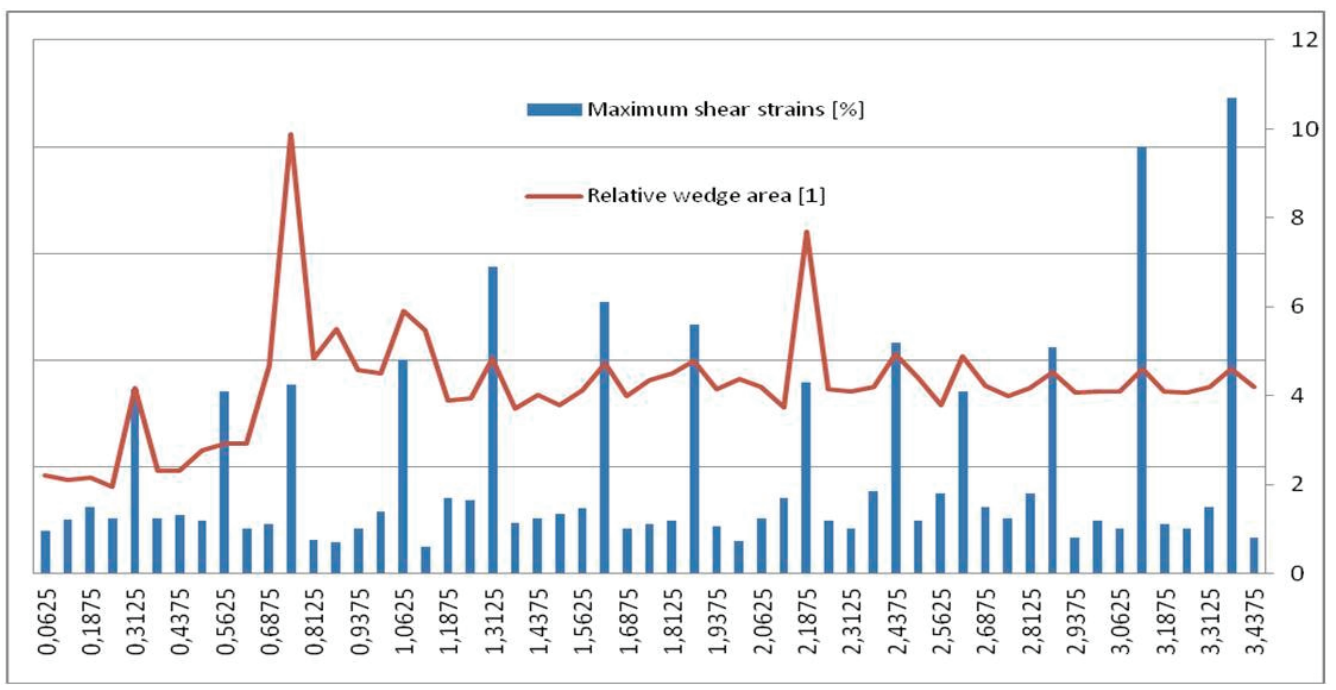

Fig. 10. Deformation cycles for the test on rigid wall moving out of the granular material, visible in maximum shear strains and estimated area of active soil wedge.

Wall displacements (horizontal axis) in [mm]

The whole deformation cycle repeats itself, as can be seen in Figs. 9 and 10, which represent the whole test programme, every 4 displacement steps on average. It can be seen from Figs. 4-8 compared to Figs. 9 and 10 that localized shear band defines 


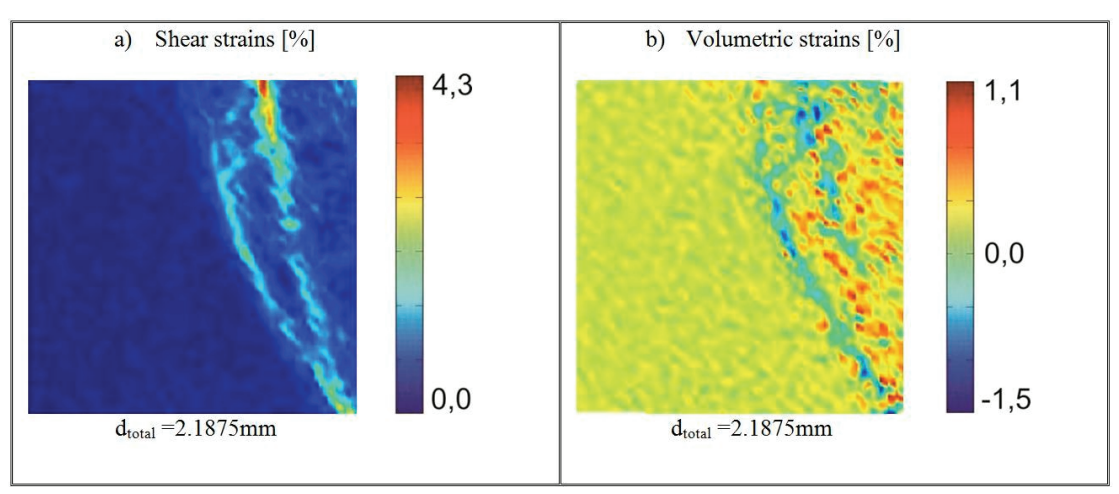

Fig. 11. Double shear band which appeared at some stage of the Test 07_05_28

bigger active soil wedge and undergoes greater strains than granular material experiencing diffused strains inside the bigger wedge. This kind of behavior was not, to our knowledge, been reported in granular science literature yet and could be observed here owing to great accuracy of the DIC (PIV) method. Beside "average" deformation cycle, represented by Figs. 4-8, some out of the common ones appeared during most of the tests, showing nonstandard failure modes, like the one visible in Fig. 11, for example, where localized shear band is doubled. Some extra-large soil wedges appeared also in some tests (see Fig. 10).

\section{CONCLUSIONS}

The experimental data presented in this paper show that the deformation of granular sample behind retaining wall can undergo some cyclic changes. The nature of the cycles is not clear - it is probably related to some micromechanical features of granular materials, which are recently extensively studied in many research centers in the world. Such cyclic behavior could presumably explain the cyclic changes of earth thrust on retaining walls, reported in a number of historical papers, but drawing any decisive conclusions needs further research. Employing very precise DIC (PIV) method can help to relate micro and macro-scale behavior of granular materials.

\section{REFERENCES}

[1] LeŚNiewsKa D., MuIR WoOd D., Observations of stresses and strains in a granular material, J. Engineering Mechanics, ASCE, 2008, 135, 9, 1038-1054.

[2] LeŚniewska D., Muir Wood D., Pietrzak M., Particle scale features in shearing of glass ballotini. Powders and Grains, (ed.) M. Nakagawa, S. Luding, New York, AIP Conference Proceedings, 2009, Vol. 1145, 335-338. 
[3] LeŚniewsKa D., Muir Wood D., Photoelastic and photographic study of a granular material, Geotechnique, 2010, 60, 903-911.

[4] Muir Wood D., LeŚNIEWSKA D., Stresses in granular materials, Granular Matter, 2011, 13, 395415.

[5] Niedostatkiewicz M., LeŚNiewSKa D., TejChMAn J., Experimental analysis of shear zone patterns in sand during earth pressure problems using Particle Image Velocimetry, Strain, 2011, 47, 218-231.

[6] Widuliński Ł., Tejchman J., Kozicki J., LeŚniewsKa D., Discrete simulations of shear zone patterning in sand in earth pressure problems of a retaining wall, Int. J. Solids Struct., 2011, 48, 11911209.

[7] White D.J., TAKe W.A., GeoPIV; Particle image velocimetry (PIV) software for use in geotechnical testing. Manual for GeoPIV, Technical Report D-SOILS-TR322, Cambridge University Engineering Department, 2002.

[8] White D.J., TAKe W.A., Bolton M.D., Soil deformation measurements using particle image velocimetry (PIV) and photogrammetry, Geotechnique, 2003, 53, 619-631.

[9] White D.J., RANDolPh M., Thompson B., An image-based deformation measurement system for the geotechnical centrifuge, Int. J. Phys. Model. Geotech., 2005, 3, 1-12. 